\title{
The Continuum Model: Statistical Implications
}

\author{
STEPHEN COOPER \\ Department of Microbiology and Immunology, University of \\ Michigan Medical School, Ann Arbor, Michigan 48109, U.S.A.
}

(Received 10 July 1981)

\begin{abstract}
The Continuum Model postulates that preparations for the initiation of DNA synthesis takes place continuously, and in all phases of the cell cycle. There are no G1-specific events involved in the initiation of DNA synthesis. The statistical predictions of the Continuum Model are now presented with four basic variables: (1) the rate of initiator synthesis, (2) the time for passage through the replication-segregation sequence, (3) the amount of initiator required for initiation of DNA synthesis in a particular cell, and (4) the variation in equipartition of cells at division. Computer simulations reveal that the Continuum Model is consistent with both $\alpha$ and $\beta$-curves, as well as the quartile test for $\beta$-curves. It also explains sister-sister correlations, and the corrclations between cell mass at various times in the division cycle and cell interdivision times. With one additional parameter, the Continuum Model can also explain mother-daughter correlations. The Continuum Model accounts for the statistical data which has previously been used to support the Transition-Probability Model. It has a simple biochemical basis, and can explain the observed biochemical and biological observations of cell growth and division.
\end{abstract}

\section{Introduction}

In this paper the Continuum Model will be shown to explain the wide range of experimental data on cell division. The G1 period, the period between cell division and the start of DNA synthesis, has generally been thought to contain the crucial events which are involved in the regulation of cell growth. Support for this idea has come from a number of diverse experimental approaches-biological, biochemical, genetic, and to a large measure, statistical.

Alternatively, the Continuum Model proposes that there are no G1specific events. Events which regulate the rate of cell proliferation are occurring during all phases of the division cycle. The biological, biochemical and genetical experiments have been analyzed (Cooper, 1979, 1981a, b) and shown to be consistent with the Continuum Model. The large amount of statistical data on cell growth and interdivision times has led to the formulation of a particular G1-regulation model, the Transition-Probability 783 
Model (Smith \& Martin, 1973). I will now show that the Continuum Model is consistent with this large mass of statistical data on cell division.

I shall begin with a description of the Continuum Model. This will be followed by a description of the current G1 model and the application of the Continuum Model to the understanding of the various types of experimental support for the G1 model. After this review I will describe the basic ideas behind the Transition-Probability Model, and then apply the Continuum Model to the data which has been presented in support of the Transition-Probability Model.

\section{The Continuum Model}

The Continuum Model proposes that the processes involved in the regulation of the initiation of DNA synthesis occur throughout the division cycle, and in all phases of the division cycle. Once DNA synthesis is initiated, preparations for the next initiation are started immediately. Thus, preparations for DNA synthesis are continuous. The Continuum Model implies that there are no G1-specific functions involved in the initiation of DNA synthesis. Specifically, it is the rate of accumulation of a "initiator"between the starts of DNA synthesis-that regulates the frequency of initiation of $S$ phases. This in turn regulates the frequency of cell division. The rate of initiator synthesis throughout the division cycle is the major determinant of the cellular interdivision time. A G1 period is found when the time between initiations of the $S$ phase is greater than the sum of the $\mathrm{S}, \mathrm{G} 2$, and $\mathrm{M}$ periods. Because cells growing exponentially exhibit a relatively small change in the $S$ and G2 periods with changes in growth rate (Coopcr, 1979, 1981a; Cameron, 1977), cells with large interdivision times have large $G 1$ periods and cells with small interdivision times have small $\mathrm{G} 1$ periods.

The Continuum Model was developed as a unified model for the division cycles of both prokaryotes and eukaryotes (Cooper, 1979). For a long time the main distinction between these groups (with regard to DNA synthesis during the division cycle) was that the eukaryotes exhibited a G1 period, while the prokaryotes did not. This distinction was blurred as later experiments demonstrated that some eukaryotic cells did not have a G1 period and that prokaryotes can have a G1 period. The Continuum Model was developed and proposed as a unified description explaining the regulation of both types of cells and the different patterns of DNA synthesis (Cooper, 1979). In explaining the different patterns of DNA synthesis it was noted that the different patterns observed are part of a continuum of patterns. Variations in rate of initiator synthesis relative to the $S$ and G2 periods 
produce the different patterns of DNA synthesis observed during the division cycle.

The exact nature of the molecules involved in the regulation of DNA synthesis, and their mode of synthesis and accumulation is not crucial for the proposed Continuum Model. It should merely be noted that the preparation for DNA synthesis is viewed as an accumulation process, and that when a required amount of initiator has accumulated, DNA synthesis starts. The formal details of this model have been previously presented (Cooper, $1981 a$ ). It has also been argued that the initiator is protein, or a relatively constant fraction of protein, or is synthesized in a manner similar to protein (Cooper, 1981b).

An important corollary of the Continuum Model is the proposition that the act of cell division is the end of a sequential process (S, G2 and M) and the beginning of no processes. This idea, termed the Central Dogma of Cell Biology (Cooper, 1981 b), may explain why there are no G1 specific events. Because a G1-specific event must occur after cell division and before $\mathbf{S}$, it follows that if there is no information from cell division to the cell substance that a cell has divided, then there is no way for a cell to know when to initiate a presumptive G1-specific event. Furthermore, if cell division does not affect the regulation of cell division or biosynthetic processes of the cell, it is clear that the processes begun in one cell cycle can be continued and carried to fruition within a subsequent division cycle.

\section{The G1 Model of Regulation}

Two types of experimental results have suggested the existence of G1specific events. The first is the observation that the variability in the length of time between cell divisions is primarily accounted for by the variability in the G1 period, with the $S, G 2$ and $M$ periods remaining fairly constant (Cooper, 1979). It was generally assumed that changes in the rate of passage through G1 were indications of the rate at which G1-specific event(s) were occurring, and that the shorter G1 periods produced faster growing cells.

The second type of experimental support for the existence of $\mathrm{G} 1$ regulatory functions is the ubiquitous finding that cells appear to be "arrested in the G1 period" under a variety of conditions which inhibit growth. The cessation of growth can be the result of the addition of inhibitors, starvation for required media constituents, or the result of various conditional mutations. (See Cooper (1981a) for an extensive review.) The observation of "G1-arrested" cells was interpreted as indicating that cells can come to rest at specific G1-arrest points. These arrest points were thought to imply the existence of $\mathrm{G} 1$-specific functions. 


\section{Reanalysis of the G1 Model}

I will briefly reinterpret the two major supports for the G1 model according to the Continuum Model. The variability of the G1 period is merely a consequence of the observed constancy of the $S$ and G2 periods. If one varies the division cycle over a wide range of times, and keeps the $S$ and $G 2$ periods constant, the G1 period will have to be the most variable period. Rather than the G1 period varying and producing the observed interdivision times, the interdivision times produce the observed G1 period. The main variable determining the interdivision time is the time between initiations of DNA synthesis. There is no way to choose between the Continuum Model and the G1 model in this respect, as all of the data for eukaryotes is consistent with both viewpoints. When one looks at the prokaryotes, however, the situation may be more decisive. In prokaryotes, it is generally observed that there can be cells with no G1 period, and DNA synthesis can begin prior to cell division (a negative G1 period(?)). In this case the Continuum Model, not the G1 model, explains the regulation of cell proliferation and the appearance of a G1 period with a decrease in growth rate.

The analysis of G1-arrest by the Continuum Model is equally simple. When cells are placed under various deleterious conditions which arrest growth, the cells eventually produced have a G1 content of DNA. This has been interpreted, according to the G1-model, as indicating that there are various specific steps or points in the G1 period which are involved in the preparations for DNA synthesis, and when these steps are inhibited the cells come to rest at the inhibited point in G1. The Continuum Model explains G1-arrest by noting that the conditions which produce the phenomenon are generally those which inhibit protein synthesis (whether by the use of inhibitors, conditional mutants, starvation, etc.) and while initiation of DNA synthesis ceases, the progress of cells through S, G2 and $M$ does not. What is produced after a period of time, are cells (originally in the G1 period) which have been stopped with a G1 DNA content by being prevented from initiating DNA synthesis, or cells which have a G1 DNA content by virtue of having passed through the $S, G 2$ and $M$ periods producing two cells which have G1 DNA contents. The production of cells with a G1 DNA content is due to the fact that most conditions which affect cell growth stop initiation of DNA synthesis but do not affect the passage of the cells through the $S, G 2$ and $M$ periods. The $S, G 2$ and $M$ periods shall be referred to collectively as the replication-segregation sequence.

It now becomes important to show that the Continuum Model can explain the statistical data on the division cycle. The statistical data comes primarily 
from measurements of the duration of interdivision times using time lapse cinematography. Among the major findings are that there is a large amount of variability in the interdivision times of presumably homogeneous cells (Sisken \& Morasca, 1963; Cook \& Cook, 1959; Kubitschek, 1962), the interdivision time distribution is skewed to the right toward higher interdivision times, and there is a positive correlation between sister cell interdivision times (Powell, 1958; Brooks, Bennett \& Smith, 1980; Shields, 1978). There have been both positive and negative correlations reported between the interdivision times of mother and daughter cells (Miyamoto, Zeuthen \& Rasmussen, 1973; Hemon et al., 1978; Van Wijk \& Van der Poll, 1979).

\section{The Transition-Probability Model}

The most prominent model explaining the significance of the variability of cell cycle length of cells in a culture, is the Transition-Probability Model of Smith \& Martin (1973). (Others have independently proposed a similar model, Burns \& Tannock, 1970; Liebowitz \& Rubinow, 1969.) This model proposes that the cell cycle consists of a fixed period which is the same for all members of a population, and a variable period which is distributed according to a negative exponential function among the individual cell cycles. The variable part is contained entirely within $\mathrm{G} 1$ and accounts for the observed variability of the G1 period described above. The constant period is presumed to be associated (although not necessarily) with the relatively constant replication-segregation sequence. The variable portion of the cycle is assumed to result from a single, first order, random process that terminates the variable part of the cell cycle with a constant probability. This model predicts that the $\alpha$-plot of the interdivision times-i.e. a plot, on semilogarithmic paper of the fraction of cells which have not yet divided by a particular time against that time-produces a flat portion with a sharp break to a straight line with a specific slope (Fig. 1). While the data for the longer division times appear to fit the tail of the proposed graph, there is a deviation for the shorter division times. Rather than a sharp break in the $\alpha$-curve a gradual curvature is observed with the tail of the graph approaching a straight line on the semilogarithmic plot (Shields, 1977).

The original model has been modified in various way to improve the fit of the data to the model. For example, Svetina (1977) and Svetina \& Zeks (1978) proposed that a better fit was obtained if there was a continuously increasing decay constant. Further study of the relationship of cell size to interdivision time has revealed that if there is a transition probability which 


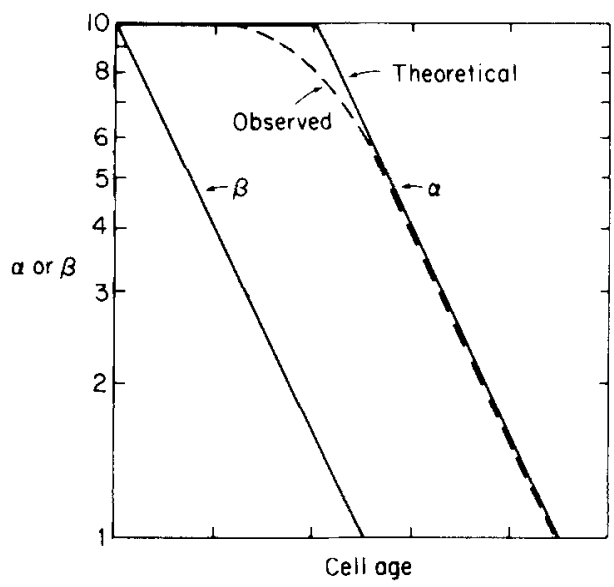

FIG. 1. Ideal $\alpha$ - and $\beta$-curves according to the Transition-Probability Model (Smith $\&$ Martin, 1973). The type of result which has been observed in published work is indicated by the dashed line.

governs division, then it must be somewhat variable and dependent on the size of the cell (Shields et al., 1978). A similar argument was made by Pardee, Shilo \& Koch (1979) who proposed that variation of both the constant period and the probability constant would give a proper fit to the observed data. Koch (1980) has pointed out that some of the fit of the data to the model may be due to a slight heterogeneity of the cells, and Castor (1980) has noted that other types of models, some of which have deterministic components may fit the data better than the TransitionProbability Model.

Minor \& Smith (1974) proposed that there was some variation in the "constant" period and that this variability eliminated the expected discontinuity in the $\alpha$-plots. They developed the $\beta$-plot, which is a plot on semilogarithmic paper of the percentage of sister-sister cells having a smaller difference in their ages at division than the time on the abscissa. The Transition-Probability Model predicts a straight line starting at $100 \%$ at time zero, which is parallel to the tail of the $\alpha$-curve (Fig. 1). A recent modification of the Transition-Probability Model proposed that the variability of the "constant" phase in a culture was due to a second random transition in the cell cycle which would produce "constant" phase variability although sister cells would have the same "constant" phase. This provides an explanation for the positive sister-sister correlation (Brooks et al., 1980).

Another test of the Transition-Probability Model is the "quartile test" proposed by Shields (1978). In this test, the sister-sister differences are grouped into four quartiles according to the shorter interdivision time in 
the sister pair. This produces four separate $\beta$-curves. The TransitionProbability Model predicts that they will be parallel and exponential with no sign of curvature. This appears to be the case in the few examples which have been published (Shields, 1978). Shields (1978) has suggested that other models produce to lines with initial curvature and that only the Transition-Probability Model gives straight $\boldsymbol{\beta}$-curves.

\section{The Statistical Continuum Model}

It is postulated that there are four basic sources of variability which combine to produce the final distribution of interdivision times. These are assigned Greek letters indicating the mean and variance of a particular variable. The four variables are: the rate of synthesis of initiator $(\kappa)$, the amount of initiator required to initiate DNA synthesis $(\mu)$, the time for passage of the cell through the replication-segregation sequence $(\rho)$, and the variability in the partition of material between the two daughter cells at division $(\delta)$. The operation of these four variables according to the

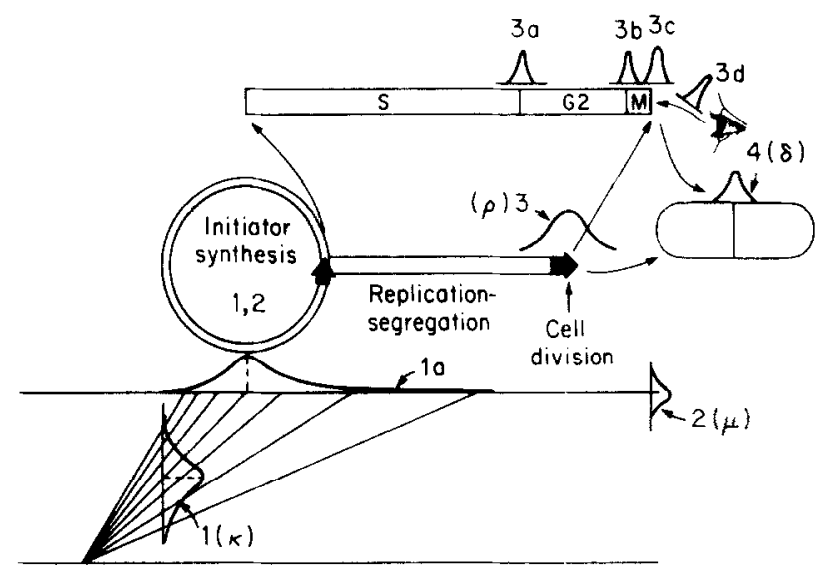

Frg. 2. Origin of statistical variation according to the Continuum Model. The central portion is the diagram of the Continuum Model with the two major aspects, initiator synthesis and the replication-segregation sequence. At the bottom is the effect of a normal distribution of initiator synthetic rates on the time required between initiations. Note that the normal distribution of rates (1) leads to a reciprocal normal distribution of initiation times (1a), which is modified by the variation in the amount of initiator required in a particular cell for initiation (2). Once initiation occurs then passage through the replication-segregation sequence follows and it is assumed to be normally distributed (3). This sequence can be subdivided into four parts which may, if desired, be considered separately. These are the $S$ period (3a), the G2 period ( $3 \mathrm{~b}$ ), the $\mathrm{M}$ period ( $3 \mathrm{c}$ ), and the variance which can be attributed to observer error in deciding when a cell has actually divided (3d). At this time only the aggregate variation (3) has been considered. Finally, the variance produced by unequal division must be considered (4). The different variables $(1-4)$ have been assigned Greek letters: $\kappa, \mu, \rho$ and $\delta$. 
Continuum Model is illustrated in Fig. 2. For simplicity, consider a collection of cells all of which have just initiated DNA synthesis with exactly the same amount of initiator. The next initiation will occur when each of the cells accumulates another unit of initiator. If the rate of synthesis is normally distributed (1), the cells will accumulate a given amount of initiator according to a reciprocal-normal distribution. Since the mass at initiation is also proposed to have a normal variation about a mean (2), there is a modified reciprocal-normal distribution (1a) of times at which initiation occurs (Kubitschek, 1971). This is followed by passage through the replicationsegregation sequence composed of S, G2, and M(3) leading to the production of two cells. The distribution of times at which division occurs is thus determined by the three variables, $\kappa, \rho$, and $\mu$.

It should be noted that the observation of when cells divide is not necessarily the same as when cells "truly" divide. This variation can be included as part of the replication-segregation sequence (variable $3 \mathrm{~d}$ ). Additional variability is introduced because cells do not distribute their mass absolutely equally at division. There is a normal distribution (4) about equal division. This final variable $(\delta)$ will introduce variation in the next division cycle.

The actual calculation of cellular interdivision times is illustrated in Fig. 3. Consider a mother cell and two daughter cells. Consider that within a mother cell division cycle the synthesis of DNA has been initiated at some initiation mass $\left(I_{I 1}=\mu_{1}\right)$, and initiator continues to accumulate during the replication-segregation sequence at a rate, $\kappa_{1}$, for a time $\rho_{1}$. For exponential synthesis of initiator during the division cycle, the amount of initiator at division $\left(I_{D 1}\right)$ of the mother cell is given by $\mu_{1} \cdot 2^{\left\langle\kappa_{1} \cdot \rho_{1}\right)}$. The cells now divide with the initiator distributed to the two newborn cells according to the variables $\delta_{2}$ and $\delta_{3}$ so that the initiator in the two new-born daughter cells is now $I_{B 2}=\delta_{2} \cdot I_{D 1}$ and $I_{B 3}=\delta_{3} \cdot I_{D 1}$. The G1 periods may now be calculated by the formulae $\mathrm{G} 1_{2}=\ln \left(\mathrm{I}_{\mathrm{I} 2} / \mathrm{I}_{\mathrm{B} 2}\right) / \ln 2 \cdot \kappa_{2}$ and $\mathrm{G1}_{3}=$ $\ln \left(\mathrm{I}_{13} / \mathrm{I}_{\mathrm{B} 3}\right) / \ln 2 \cdot \kappa_{3}$, obtained by solving for the time required to increase the initiator in the newborn cell to the amount of initiator required for initiation of DNA synthesis. Add to this G1 period the value for the replication-segregation time in that cell $\left(\rho_{2} ; \rho_{3}\right)$ and one obtains the final interdivision times (IDT). By solving for the total IDT in terms of the four basic variables one can obtain the two basic equations:

$$
\mathrm{IDT}_{2}=\left(\ln \left(\mu_{2} / \delta_{2} \cdot \mu_{1} \cdot 2^{\left(\kappa_{1} \cdot \rho_{1}\right)}\right) / \ln 2 \cdot \kappa_{2}\right)+\rho_{2}
$$

and

$$
\mathrm{IDT}_{3}=\left(\ln \left(\mu_{3} / \delta_{3} \cdot \mu_{1} \cdot 2^{\left(\kappa_{1} \cdot \rho_{1}\right)}\right) / \ln 2 \cdot \kappa_{3}\right)+\rho_{3}
$$




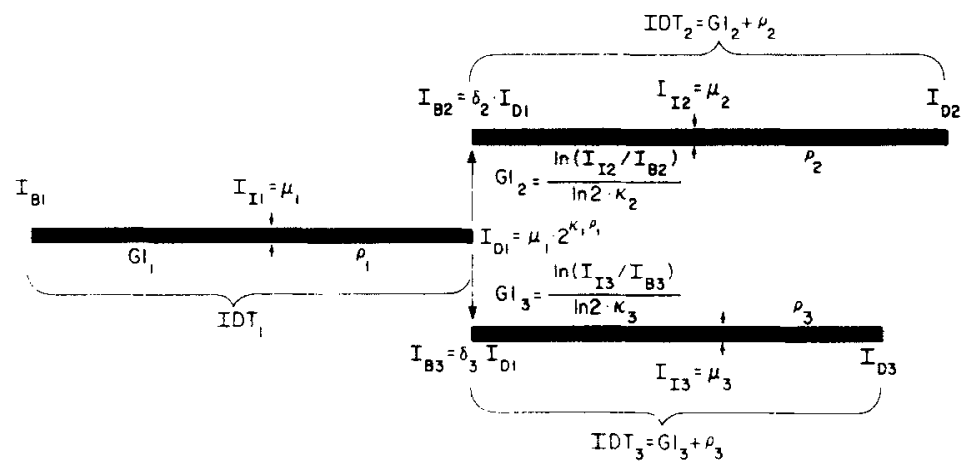

FIG. 3. Calculation of stochastic variation of interdivision times. Three different interdivision times (IDT's) are illustrated by the three dark bars. The mother cell (IDT ${ }_{1}$ ) divides to produce two daughter cells (IDT 2 and $\mathrm{IDT}_{3}$ ). The amount of initiator (I) in each of the cells at the times of birth, initiation of DNA synthesis, and division are indicated by the appropriate subscripts $\left(I_{B 1}, I_{11}, I_{\mathfrak{D} 1}, I_{B 2}, I_{12}\right.$, etc.). The Greek letters indicate the four variables which produce the observed variation in the interdivision times of the cells. $\rho$ is the mean and associated variance of the replication-segregation sequence, $\kappa$ is the mean and variance in the rate of synthesis of initiator, $\mu$ is the mean and variance in the amount of initiator required for initiation, and $\delta$ is the variation in the division process. The $\delta_{2}$ and $\delta_{3}$ are assigned mean values of 0.5 with the variance depending on the simulation. The $\delta_{2}$ and the $\delta_{3}$ (in any single division) add up to 1.0 thus producing two cells which together have the total mass of the mother cell. By convention the $\mu$ is assigned a mean value of $1 \cdot 0$. By assigning $\alpha$ a mean value of 0.01 , cell interdivision times are produced which have a mean value of approximately 100 time units (minutes, hours, etc.) and the mean value of the replication-segregation sequence can vary to produce any desired GI time. Thus, a $\rho$ with a mean value of 100 will be a cell with no measurable $G 1$, and a $\rho$ of 50 will have half of the interdivision time occupied with G1. One can have a cell with a $\kappa$ of 0.01 and a $\rho$ of 50 (thus having half of the IDT being the $\mathrm{G} 1$ period) lose its $\mathrm{G} 1$ in two ways; either by increasing the mean of the $\rho$ to 100 or by increasing the mean rate constant, $\kappa$, to 0.02 producing cells with interdivision times of 50 time units. The calculations presented here are only those for IDT $_{2}$ and IDT 3 which are sister cells. The results from either cell can give the proper $\alpha$-plot, and the difference between the two are the data for the $\beta$-plot.

Consider a special case in which the values for $\kappa, \rho, \mu$, and $\delta$ are $0 \cdot 01$, 50,1 and 0.5 respectively. In this case the mother cell initiates DNA synthesis at precisely fifty minutes prior to division with an initiator mass of $1 \cdot 0$, and the amount of initiator at division is $2^{(0.01 \times 50)}=2^{0.5}=1 \cdot 41$. The two equal daughter cells then have 0.0705 units of initiator. The G1 is equal to $\ln (1 \cdot 0 / 0 \cdot 705) / \ln 2 \cdot(0 \cdot 01)$ which is 50 . To this $\mathrm{G} 1$ period of 50 is added a replication-segregation sequence of 50 units to give a total interdivision time in the two daughters of 100 . The actual simulation repeats this calculation for groups of up to 400 mother cells with different sets of variables, chosen using a random normal distribution function, according to the input means and variances. The results are the IDT's of the daughter cells. This data is then used to generate the desired plots and correlations. 


\section{Simulation Results}

It is impracticable to present the general results of the simulations. This can be seen by considering that there are five independent variables (the four variances and the possible variation of the fraction of the total interdivision time that is taken up by the replication-segregation sequence), and if simulations were performed in all combinations for five values of variables, there would be $5^{5}$ or 3125 different simulations. Each of these simulations would in turn have a number of output graphs and correlation values to consider. The approach taken here is to present some typical, albeit selected, simulation results, and to show that the results are quite similar to the data presented in the literature. All that follows is typical of the results obtained, and no effort has been spent in showing particularly unique results.

$\alpha$-curves: The results of a number of typical simulations are presented in Figs 4 and 5, with the input parameters and the output results of the particular simulations presented in Table 1 . The $\alpha$-curves are similar to those which have been presented in the literature. All of the curves presented fit the general criterion that they avoid extreme values of the coefficient of variation of interdivision times. Experimental values less than $10 \%$ and greater than $30 \%$ are usually not found. The tails of the $\alpha$-curves

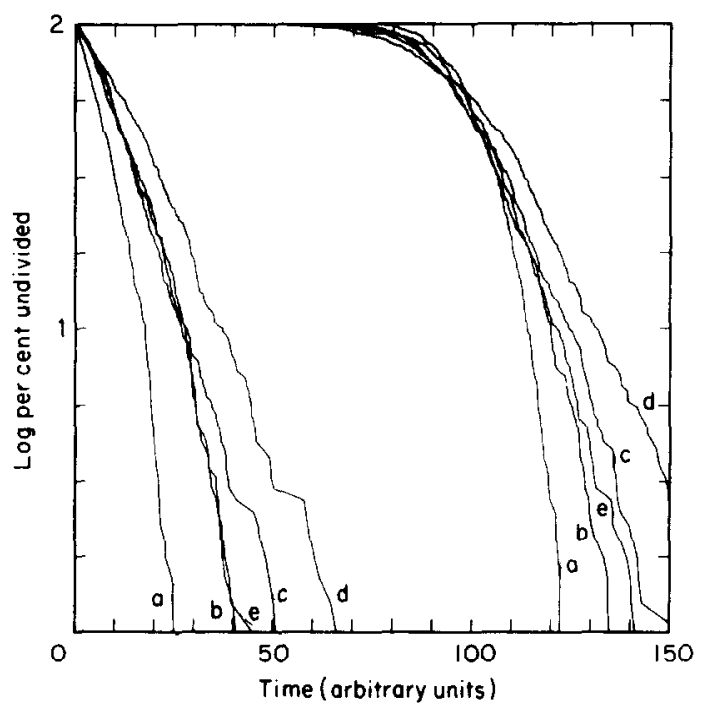

FIG. 4. $\alpha$-and $\beta$-curves for five typical simulations. The experiments are those described in Table 1, a-e. 


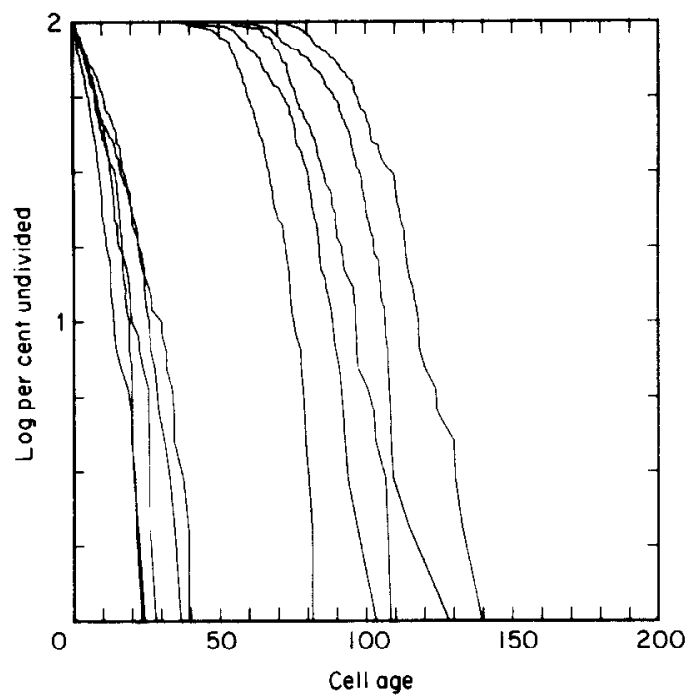

FIG. 5. A simulation in which all of the variables have some variance. The data are for experiments $\mathrm{f}-\mathrm{j}$, Table 1 .

are relatively straight and the curvature found is similar to that observed in published results (Fig. 6).

$\beta$-curves: The $\beta$-curves shown in the Figs 4 and 5 are also quite similar to the actual data found in numerous publications. Although it has been suggested that the experimentally produced $\beta$-curves are straight and that only the Transition-Probability Model will produce straight $\beta$-curves, I note that the simulated curves cannot be distinguished from straight lines (Fig. 6). This is also illustrated in Fig. 7 where it is shown that a line which does not appear to go through the origin when all of the points are plotted, does pass through the origin when the data are grouped and an artist draws a line through the data points. It should be noted that $\beta$-curves which are not straight have been reported in yeast (Fantes, 1977).

Quartile Test: The results of the quartile test for one of the simulations (c of Table 1) is shown in Fig. 8. Note that the curves of this simulation (and in fact of all simulations that do not produce too extreme values for the results) produce quite parallel, and straight, $\beta$-curves for the four quartiles. Although it may be true that only the transition probability model predicts absolutely straight and parallel beta curves for the four quartiles, it is also true that for the results normally obtained (usually with less than 400 cells in a sample) it is not possible to use the quartile test to eliminate other models of the cell cycle, and specifically the Continuum Model. If 


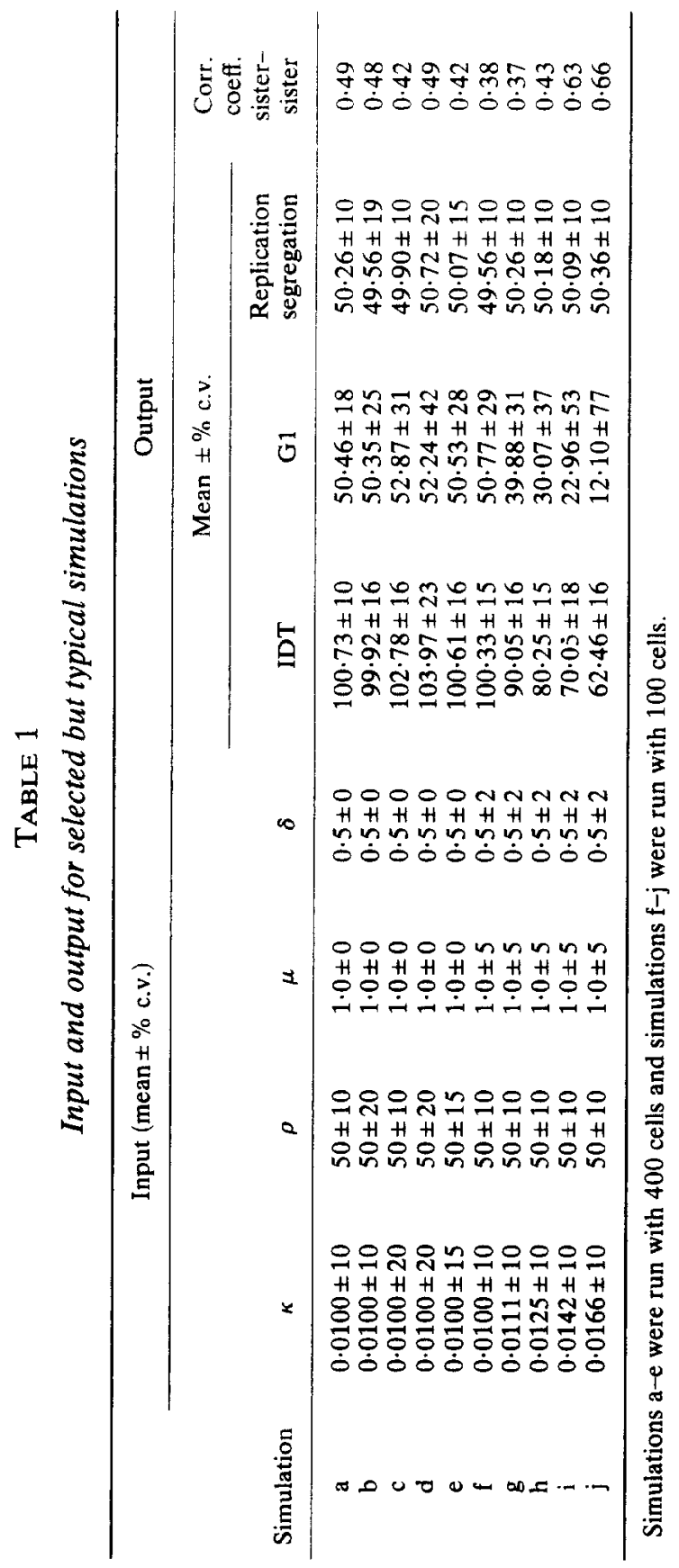




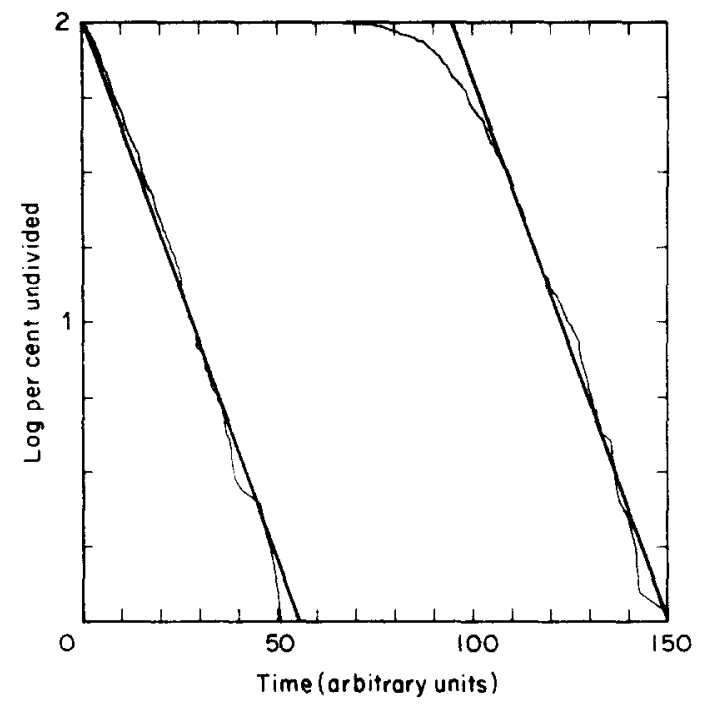

Fig. 6. Comparison of $\alpha$ - and $\beta$-curves with straight lines. The curves plotted are those from experiment $c$ of Table 1 and Fig. 4. An artist drew a best fit straight line by eye.

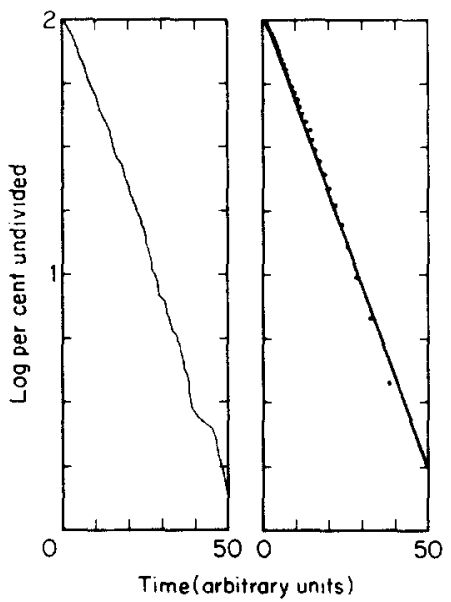

FIG. 7. Comparison of grouped data and ungrouped data. The data from experiment $c$ is plotted on the right with all 400 points connected by straight lines. At the left every 12 th data point is plotted as a point and an artist drew the best straight line. 


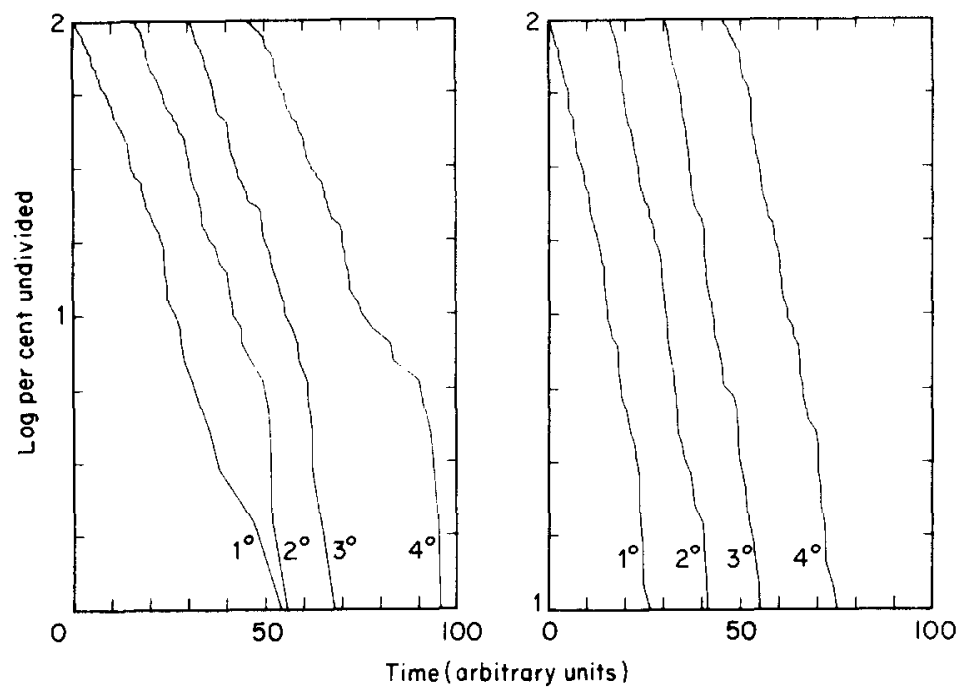

FIG. 8. The quartile test. The data from experiment c, Table 1, are arranged in four $\beta$-curves according to the age of the shorter of the two sisters. The curves are displaced to the right at 15 time unit intervals. The data are plotted down to the $1 \%$ level in (a) and to the $10 \%$ level in (b).

one plots the four $\beta$-curves down to the $10 \%$ level (Fig. 8, left) rather than the $1 \%$ level the lines appear even straighter and more parallel than otherwise.

\section{Correlations between Interdivision Times}

Sister-Sister Correlations: As seen in Table 1, and in a typical result as shown in Fig. 9, there is a significant correlation between sister cell interdivision times. That this is expected can be seen either by considering equations (1) and (2) above where it is seen that both equations have three variables $\left(\mu_{1}, \kappa_{1}\right.$, and $\left.\rho_{1}\right)$ in common, or by observing that since the time of division occurring after the replication-segregation sequence of the mother cell lengthens or shortens the daughter interdivision times by equivalent amounts this should produce a positive sister-sister correlation. Although sister-correlations may be produced by ad hoc considerations (Shields, 1978; Castor, 1980), in this case the ubiquitous finding of a positive sister-sister correlation falls naturally out of the simulation.

One of the more interesting ideas to come out of the analysis described here is an understanding of what may be called "spurious correlation" (Powell, 1958). This is the idea that a positive correlation between sister 


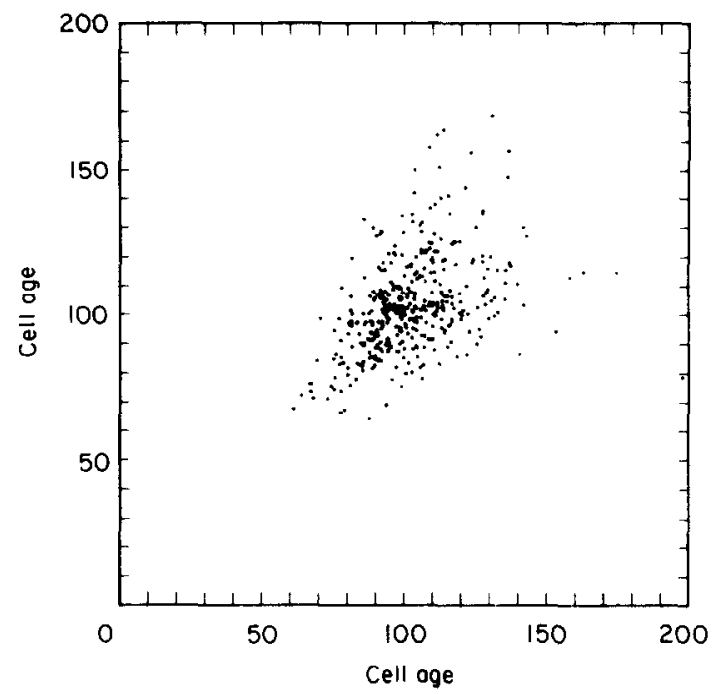

FIG. 9. A typical scatter diagram of the interdivision times of sister cells. The data are from experiment $\mathrm{c}$ in Table 1 .

cells can result from the observer accidentally reading the time of division of the mother cell too early or too late. This "error" is now included as a natural part of the replication-segregation sequence. Any imperfection in reading the time of division, rather than being a factor in generating a spurious correlation, may merely be considered to have increased, albeit slightly, the variance of the replication-segregation sequence, and it is this total variance (relative to the other variables) which contributes to the sister-sister correlation.

Mother-Daughter Correlation: Both positive and negative correlations between mother interdivision times and daughter interdivision times have been noted. If there were no mechanism to generate any positive correlation between successive generations, it would be expected that the variance of the replication-segregation sequence would generate a negative motherdaughter correlation. This is because mother cells with a shorter replicationsegregation sequence than average generate shorter mother interdivision times and simultaneously longer than expected daughter interdivision times. Given any variance in the replication-segregation sequence, if absolutely no correlation between mother and daughter interdivision times was observed experimentally then the Continuum Model would suggest that in fact there was a mechanism which was working to produce a positive correlation between generations with the two positive correlations cancelling out. How then, can one explain the accumulating evidence for the 
existence of positive mother-daughter correlations (Miyamoto, Zeuthen \& Rasmussen, 1973; Van Wijk \& van de Poll, 1979; Hemon et al., 1978)? Consider that the values for the rates of initiator synthesis are not independent in different generations. That is, a cell with a relatively rapid synthesis of initiator produces daughter cells which have a greater probability of having an elevated rate of initiator synthesis. On a biochemical level this may be considered due to something about the cytoplasm or the surface being more "active" than average, and that this property is carried forth by the two daughter cells. (A modification of the current program to simulate this "autocorrelation" between generations is being developed.) This will lead to a positive correlation between the mother and daughter interdivision times, in spite of the propensity for the variation in the replication-segregation sequence to produce a negative mother-daughter correlation. Thus, it is possible to imagine, according to the Continuum Model, how either positive or negative mother-daughter correlations can be explained. The Transition-Probability Model does not predict or explain any positive correlations between the generations (Brooks et al., 1980).

\section{Cell Size and Interdivision Time}

There have been many studies supporting the role of cell size as a primary control of cell division (Fantes, 1977; Nurse, 1975; Alberghina \& Sturani, 1981). The Transition-Probability Model predicts a particular distribution of interdivision times, but makes no predictions regarding correlations or relations between cell size and cycle times. In contrast, the Continuum Model makes very clear predictions regarding the correlation between cell size and cycle times. Essentially all of the predictions that are made are consistent with observations reported in the literature. For example, Prescott (1956) found a negative correlation between birth weight and generation time in Amoeba proteus, a relationship which is predicted by the Continuum Model and supported by the results of simulation studies. It is always found that there is a negative correlation between the amount of initiator at birth and the interdivision time of a cell.

Koch \& Schaechter (1963) have observed that the coefficient of variation of size at division is less than the coefficient of variation of cell interdivision times. This, too, is observed in simulations described here. It has also been found that the variability of cell volumes at initiation of DNA synthesis (in bacteria) is considerably less than the variability of the cell volumes at division (Koppes, Woldringh \& Nanninga, 1978; Nanninga, Woldringh \& Koppes, 1981). This is also seen to be the case in simulation studies. Thus 
the Continuum Model not only fits the observations on cell cycle distributions, but the relationship of these distributions to cell size or mass.

\section{Discussion}

Is cell regulation deterministic or probabilistic? This question, which has been asked many times before, is clarified by the discussion of the Continuum Model presented here. The Continuum Model is a deterministic model, but even a deterministic model may have probabilistic components. In biological systems which are the result of complex biochemical interactions it is clear that at some level the deterministic features of the system are lost and replaced by more probabilistic features. At the molecular level the reactions are clearly probabilistic. The sum of the reactions do combine, however, to produce a deterministic result. Any deterministic model must have some variability and it is this variability which has been the subject of this paper.

The Continuum Model is a simple model which is able to explain a large array of experimental data on the regulation of cell division and the cell cycle. While the statistical arguments presented here may not be able to absolutely confirm the model (as these arguments are not able to eliminate other descriptions which fit the data) the fact that the Continuum Model fits a large array of data should not be overlooked. The Continuum Model covers both the eukaryotes and prokaryotes, and can explain steady state as well as arrested growth situations.

Jim Hill, an undergraduate at the University of Michigan, was instrumental in getting the program in a form in which large numbers of simulations could be run efficiently and quickly. He has also prepared the program and accompanying instructions which will allow anyone in the world to use it, either by using the Michigan Terminal System directly (in North America) or by using Telenet (in Europe and other areas). Ken Guirre of the Department of Biostatistics introduced me to the amazing capabilities of MIDAS and allowed me to get the programming done in the most efficient manner. Funds for the Computing time were supplied by the Computer Committee of the University of Michigan Medical School, and their generous support is hereby acknowledged. Anna Frame's artistry is evident in the figures, and Alexandra Cooper's editing skills are evident in whatever clarity is in the text.

\section{REFERENCES}

Alberghina, L. \& Sturani, E. (1981). Micro. Rev. 45, 99.

BroOks, R. F., BENNETT, D. C. \& SMITH, J. A. (1980). Cell 19, 493.

Burns, F. J. \& TANNOCK, I. F. (1970). Cell Tissue Kinet. 3, 321. 
CAMERon, I. L. (1971). Cell proliferation and renewal in the mammalian body. In: Cellular and Molecular Renewal in the Mammalian Body (I. L. Cameron and J. D. Thrasher eds). London: Academic Press. pp. 45-85.

CASTOR, L. N. (1980). Nature, Lond. 287, 857.

COOK, J. R. \& CoOK, B. (1962). Exp. Cell Res. 28, 524.

COOPER, S. (1979). Nature, Lond. 280, 17.

CoOper, S. (1981a). The Continuum Model: Application to G1-arrested and G(0). In: Cell Growth (C. Nicolini, ed.). New York: Plenum Press.

COOPER, S. (1981 h). Cell Binl. Int. Rep. 5, 539.

FANTES, P. A. (1977). J. Cell Sci. 24, 51.

Hemon, D., Collyn-D'Hooghe, M., Valleron, A.-J. \& Malaise, E. P. (1978). Statistical methods for the estimation and analysis of correlations between characteristics of cells observed using time-lapse microcinematography. In: Biomathematics and Cell Kinetics (Valleron, A.-J. and Macdonald, P. D. M., eds). Amsterdam: Elsevier/NorthHolland.

KoCH, A. L. \& SChaechTeR, M. (1962). J. Gen. Microbiol. 29, 435.

KuCH, A. L. (1980). Nature, Lond. 286, 80.

KoPPES, L. J. H., WOldRingh, C. L. \& NANNINGA, N. (1978). J. Bact. 134, 423.

KubitsCheK, H. E. (1962). Exp. Cell Res. 26, 439.

KUBITSCHEK, H. E. (1971). Biophys. J. 11, 961.

LeBowitz, J. \& Rubinow, S. I. (1969). J. theor. Biol. 73, 99.

Minor, P. D. \& SMiTh, J. A. (1974). Nature, Lond. 248, 241.

Miyamoto, H., Zeuthen, E. \& Rasmussen, L. (1973). Clonal growth of mouse cells (Strain L). J. Cell. Sci. 13, 379-388.

NANNinga, N., Woldringh, C. L. \& Koppes, L. J. H. (1981). Growth and division of Escherichia coli. In: Cell Growth (Nicolini, D. ed.). New York: Plenum Press.

NASMYTH, K. A. (1979). J. Cell Sci. 36, 155.

Nurse, P. (1975). Nature, Lond. 256, 547.

Pardee, A. B., ShILO, B.-Z. \& КОСH, A. L. (1979). Variability of the cell cycle. In: Hormones and Cell Culture (Sato, G. S. and Ross, R., eds). Cold Spring Harbor Biological Laboratory. pp. 373-392.

POWELL, E. O. (1958). J. Gen. Microbiol. 18, 382.

PrescotT, D. M. (1956). Exp. Cell. Res. 11, 86.

SHIELDS, R. (1978). Nature, Lond. 273, 755.

SHIELDS, R. (1977). Nature, Lond. 267, 704.

Shields, R. \& SMith, J. A. (1977). J. Cell Physiol. 91, 345.

Shields, R., Brooks, R. F., Riddle, P. N., Capellaro, D. F. \& Delia, D. (1978). Cell 15, 469.

Sisken, J. E. \& MORASCA, L. (1965). J. Cell Biol. 25, 179.

Smith, J. A. \& Martin, L. (1973). Proc. nain. Acad. Sci. U.S.A. 70, 1263.

Svetina, S (1977). Cell Tissue Kinet. 10, 575.

SVETINA, S. \& ZEKS, B. (1978). Transition probability models of the cell cycle exhibiting the age distribution for cells in the indeterministic state of the cell cycle. In: Biomathematics and Cell Kinetics (Valleron, A.-J. and Macdonald, P. D. M. eds), Amsterdam: Elsevier/North-Holland Biomedical Press, pp. 71-82.

VAN WIJK, R. \& VAN DE POLl, K. W. (1979). Cell Tissue Kinet. 12, 659. 